\title{
Disjoint pattern matching and implication in strings
}

\author{
Leonid Libkin $^{\mathrm{a}, *}$, Cristina Sirangelo ${ }^{\mathrm{b}}$ \\ a School of Informatics, University of Edinburgh, United Kingdom \\ b LSV, ENS-Cachan, France
}

\section{A R T I C L E I N F O}

\section{Article history:}

Received 8 September 2009

Accepted 21 November 2009

Available online 4 December 2009

Communicated by J. Chomicki

\section{Keywords:}

Theory of computation

Strings

Patterns

Implication

XML

\begin{abstract}
A B S T R A C T
We deal with the problem of deciding whether a given set of string patterns implies the presence of a fixed pattern. While checking whether a set of patterns occurs in a string is solvable in polynomial time, this implication problem is well known to be intractable. Here we consider a version of the problem when patterns in the set are required to be disjoint. We show that for such a version of the problem the situation is reversed: checking whether a set of patterns occurs in a string is NP-complete, but the implication problem is solvable in polynomial time.
\end{abstract}

(C) 2009 Elsevier B.V. All rights reserved.

\section{Introduction and the main result}

The problem we consider in this Note was motivated by answering queries in incompletely specified XML documents. Suppose that $\mathcal{L}$ is a set of letters, or labels, assumed to be countably infinite, and that _ is a special symbol (wildcard) not in $\mathcal{L}$. By $\mathcal{L}_{-}$we denote $\mathcal{L} \cup\left\{\left\{_{-}\right\}\right.$. A pattern is a finite string over $\mathcal{L}_{-}$. If a string $s$ over $\mathcal{L}$ matches a pattern $\pi$, we write $s \models \pi$. More precisely, if $s=a_{0} \ldots a_{n-1}$ and $\pi=b_{0} \ldots b_{m-1}$, then $s=\pi$ if there is a number $j \leqslant n-m$ so that for each $b_{i}$ that is not a wildcard, we have $b_{i}=a_{i+j}$ (i.e., a wildcard can match every symbol). In this case we also say $s$ matches $\pi$ from $j$ to $j+m-1$. If $s$ matches every pattern in a set $\Pi$ of patterns, we write $s \models \Pi$.

We say that a set $\Pi$ of patterns implies a pattern $\pi$, written as $\Pi \vdash \pi$ if $s \models \pi$ whenever $s \models \Pi$, for every finite string $s$ over $\mathcal{L}$. Now, for each pattern $\pi$, consider the implication problem $\operatorname{ImpL}(\pi)$ : its input is a set $\Pi$ of patterns, and the question is whether $\Pi \vdash \pi$.

This problem is known to be coNP-complete, even for very simple patterns $\pi$. To see hardness, consider the fol-

\footnotetext{
* Corresponding author.

E-mail addresses: libkin@inf.ed.ac.uk (L. Libkin),

cristina.sirangelo@lsv.ens-cachan.fr (C. Sirangelo).
}

lowing well-known NP-complete problem [4]: given strings $s_{1}, \ldots, s_{l}$ and a number $K$ in unary, is there a string $s$ of length $K$ so that each $s_{i}$ is a substring of $s$ ? For the reduction, assume that $\$$ is in $\mathcal{L}$ but not used in any of the $s_{i}$ 's, and take each $s_{i}$ as a pattern, as well as the string $\$ \_\ldots \_\$$ (consisting of exactly $K$ wildcards between a leading and a trailing \$-symbol). Then this set of patterns implies _\$_ iff every string that contains all the $s_{i}$ 's has length greater than $K$ (as it has symbols both left and right of a $\$$ ); this shows coNP-hardness of IMPL(_\$_).

Notice, however, that in this reduction the matches for the $s_{i}$ 's may well overlap. We are concerned with the implication when pattern matches are required to be disjoint. More precisely, we say that $s$ disjointly matches $\left\{\pi_{0}, \ldots\right.$, $\left.\pi_{n-1}\right\}$ if there are disjoint intervals $\left[i_{0}, j_{0}\right], \ldots,\left[i_{n-1}, j_{n-1}\right]$ so that $s$ matches each $\pi_{l}$ from $i_{l}$ to $j_{l}$. We denote this by $s \models_{\mathrm{d}}\left\{\pi_{0}, \ldots, \pi_{n-1}\right\}$. We then say that $\left\{\pi_{0}, \ldots, \pi_{n-1}\right\}$ disjointly implies $\pi$, written as $\left\{\pi_{0}, \ldots, \pi_{n-1}\right\} \vdash_{\mathrm{d}} \pi$, if, for every $s$, we have $s \models \pi$ whenever $s \models_{\mathrm{d}}\left\{\pi_{0}, \ldots, \pi_{n-1}\right\}$.

The disjoint implication problem $\operatorname{IMPL}_{\mathrm{d}}(\pi)$ then takes a set of patterns $\Pi$ as an input and checks whether $\Pi \vdash_{\mathrm{d}} \pi$.

The motivation for this notion comes from the study of querying incompletely described XML documents [1-3]. For instance, one may have an incomplete description of children of a node, and a query given by a pattern over 
a set of possible labels of nodes. The standard database approach is to look for certain answers, i.e., query evaluation should return true if every XML document matching the incomplete description matches the query pattern as well. This is precisely the pattern implication problem. Very often, XML documents are accessed using the DOM interface [5], in which every node has its own unique id. Then matches of different subpatterns cannot overlap, as this would lead to id clashes. Hence, query answering in incomplete XML documents with node ids corresponds to the problem of disjoint pattern implication. It is also common to consider the setting where a query is fixed, while data varies (the notion of data complexity).

For the usual matching notion, checking whether $s=\Pi$ is polynomial, but $\operatorname{IMPL}(\pi)$ could be coNP-hard, as we have just seen. For the disjoint versions of these problems, the situation is exactly the opposite: checking whether $s \models_{\mathrm{d}}$ $\Pi$ is intractable, while $\operatorname{IMPL}_{d}(\pi)$ is solvable in polynomial time for every $\pi$.

\section{Theorem 1.}

- The problem of checking, for a string $s$ and a set of patterns $\Pi$, whether $s \models_{\mathrm{d}} \Pi$ is NP-complete. It remains NPcomplete even if the patterns do not use the wildcard.

- For each pattern $\pi$, the problem $\operatorname{IMPL}_{\mathrm{d}}(\pi)$ is solvable in polynomial time.

\section{Proof of Theorem 1}

We start with the first item. NP membership is immediate - just guess a disjoint matching. For hardness, we use a reduction from the following version of binpacking: given a set $N=\left\{u_{0}, \ldots, u_{n-1}\right\} \subset \mathbb{N}$, as well as $\ell \in \mathbb{N}$ (number of bins) and $m \in \mathbb{N}$ (capacity of bins), is there a partition of $N$ into $\ell$ sets so that the sum of numbers in each set is at most $m$ ? The problem is NP-hard even if all the numbers are in unary. Now assume that 0 and 1 are elements of $\mathcal{L}$; define $\pi_{i}$ as $0^{u_{i}}$ for each $i \leqslant n-1$, and define $s=\left(0^{m} 1\right)^{\ell}$. Then $s \models_{\mathrm{d}}\left\{\pi_{0}, \ldots, \pi_{n-1}\right\}$ iff there exists a solution to the binpacking problem.

We now move to the second item. We start with some additional notations and auxiliary results.

Given a string $s$ and a position $i<|s|$, we shall refer to $i$ as the prefix of that position in $s$ and to $|s|-i-1$ as the suffix of that position. If $s=\pi$ from $i$ to $j$, we will also say that $s$ matches $\pi$ with prefix $i$ and suffix $|s|-j-1$.

We apply the notion of pattern matching, as is, to pairs of patterns: given two patterns $\pi_{1}=a_{0} \ldots a_{n-1}$ and $\pi_{2}=$ $b_{0} \ldots b_{m-1}$ in $\mathcal{L}^{*}$, we write $\pi_{1} \models \pi_{2}$ iff there is a number $j \leqslant n-m$ so that for each $b_{i}$ that is not a wildcard, we have $b_{i}=a_{i+j}$.

We also extend the notion of disjoint pattern matching to ordered sets of patterns. Given a string $s \in \mathcal{L}^{*}$, a set of patterns $\Pi=\left\{\pi_{0}, \ldots, \pi_{n-1}\right\}$ in $\mathcal{L}^{*}$, a subset $O \subseteq \Pi$, and a total order $<_{0}$ over $O$, we say that $s$ disjointly matches $\Pi$ under $<_{0}$, and write $s \models_{\mathrm{d}}\left(\Pi,<_{0}\right)$, if there are disjoint intervals $\left[i_{0}, j_{0}\right], \ldots,\left[i_{n-1}, j_{n-1}\right]$ so that:

- $s$ matches each $\pi_{l}$ from $i_{l}$ to $j_{l}$;

- for all $\pi_{l}, \pi_{r} \in O$, if $\pi_{l}<_{0} \pi_{r}$ then $j_{l}<i_{r}$.
This naturally leads to the notion $\left(\Pi,<_{0}\right) \vdash_{\mathrm{d}} \pi$.

Assume that $\pi$ is a pattern in $\mathcal{L}^{*}$. Let $\Pi=\left\{\pi_{0}, \ldots\right.$, $\pi_{n-1}$ \} be a set of patterns in $\mathcal{L}_{-}^{*}$. Without loss of generality, let

$\pi=\left({ }_{-}\right)^{k_{\text {pre }}} \pi_{c}\left({ }_{-}\right)^{k_{\text {suf }}}$

where $k_{\text {pre }}, k_{\text {suf }} \geqslant 0$ and $\pi_{c}$ is either the empty string or

$\pi_{c}=\ell_{0} \ell_{2} \cdots \ell_{p-1}$

with $\ell_{0}, \ell_{p-1} \in \mathcal{L}$ and $p \geqslant 1$.

If $\pi_{c}$ is empty, $\operatorname{IMPL}_{\mathrm{d}}(\pi)$ is straightforward: $\Pi \vdash_{\mathrm{d}}\left({ }_{(}\right)^{k}$ iff $\sum_{\pi_{i} \in \Pi}\left|\pi_{i}\right| \geqslant k$. In fact if $\sum_{\pi_{i} \in \Pi}\left|\pi_{i}\right| \geqslant k$, given a string $s$ such that $s \models_{\mathrm{d}} \Pi$ we know $|s| \geqslant \sum_{\pi_{i} \in \Pi}\left|\pi_{i}\right| \geqslant k$. Then $s \models\left({ }_{-}\right)^{k}$. On the other hand, for each $\pi_{i} \in \Pi$, by definition of the matching relation, there exists always a string $s_{i}$ such that $s_{i}=\pi_{i}$ and $\left|s_{i}\right|=\left|\pi_{i}\right|$. Then the string $s=s_{0} s_{1} \ldots s_{n-1}$ disjointly matches $\Pi$ and has size $|s|=$ $\sum_{\pi_{i} \in \Pi}\left|\pi_{i}\right|$. Thus if $\sum_{\pi_{i} \in \Pi}\left|\pi_{i}\right|<k$, clearly $s \not \models\left({ }_{-}\right)^{k}$, and therefore $\Pi \nvdash_{\mathrm{d}}\left({ }_{-}\right)^{k}$.

Hence, in the rest of the proof $\pi_{c}$ is assumed to be nonempty. Then, given a string $s$, clearly $s \models \pi$ iff $s \models \pi_{c}$ with prefix at least $k_{\text {pre }}$ and suffix at least $k_{\text {suf }}$.

We will also need the following:

Claim 1. If $O=\left\{\rho_{0}, \ldots, \rho_{m-1}\right\}$ is an ordered set of patterns (with ordering $<0$ ), then there exists a string $s$ over $\mathcal{L}$ such that $s \models_{\mathrm{d}}\left(0,<_{0}\right)$ and, if $s \models \pi$, there exists an index $i \in$ $[0, \ldots, m-1]$ so that the following hold:

- $\rho_{i} \models \pi_{c}$;

- if $i=0$ then $\rho_{i}=\pi_{c}$ with prefix at least $k_{\text {pre }}$;

- if $i=m-1$ then $\rho_{i} \models \pi_{c}$ with suffix at least $k_{\text {suf }}$.

Proof. Assume w.l.o.g. that in the ordered set 0 , we have $\rho_{i}<_{0} \rho_{j}$ whenever $i<j$. We construct the string $s$ as follows. Let $l$ be an arbitrary label of $\mathcal{L}$ not occurring in $\pi_{c}$. For each $\rho_{i}$, if $\rho_{i}=a_{0} \ldots a_{k}$, construct the string $s_{i}=b_{0} \ldots b_{k}$, where $b_{i}=a_{i}$ if $a_{i} \in \mathcal{L}$, and $b_{i}=l$ if $a_{i}$ is a wildcard. Fix arbitrarily an integer $w \geqslant\left|\pi_{c}\right|$ and define $s=s_{0} l^{w} s_{1} l^{w} \ldots l^{w} s_{m-1}$ (which gives $s=s_{0}$ in the case $m=1)$. Clearly $s \models_{\mathrm{d}}\left(0,<_{0}\right)$.

Now assume that $s \models \pi$. This implies that $s \models \pi_{c}$ from some position $i_{c}$ to some position $j_{c}$ with prefix at least $k_{\text {pre }}$ and suffix at least $k_{\text {suf }}$. Since $\pi_{c}=\ell_{0} \ell_{1} \ldots \ell_{p-1}$ with $\ell_{0}, \ell_{p-1} \in \mathcal{L}$, the following holds:

- positions $i_{c}$ and $j_{c}$ occur within some of the substrings $s_{i}$ 's; in fact $s\left(i_{c}\right)=\ell_{0}$ and $s\left(j_{c}\right)=\ell_{p-1}$ and this can occur only in the $s_{i}$ 's as all other positions have label $l$ not occurring in $\pi_{c}$.

- positions $i_{c}$ and $j_{c}$ occur in the same substring $s_{i}$ of $s$, since $w \geqslant\left|\pi_{c}\right|$.

Fix $i$, with $0 \leqslant i \leqslant m-1$, and assume that $i_{c}$ and $j_{c}$ occur in $s_{i}$; then $s_{i} \models \pi_{c}$ starting from some position $j$. As a consequence, for all $\ell_{k}$ which is not a wildcard we have $s_{i}(j+k)=\ell_{k}$. Then, by construction, also $\rho_{i}(j+k)=$ $\ell_{k}$, which shows that $\rho_{i}=\pi_{c}$ from position $j$ to position $j+\left|\pi_{c}\right|-1$. In particular, if $i=0$, then $j=i_{c} \geqslant k_{\text {pre }}$; thus 
$\rho_{i} \models \pi_{c}$ with prefix at least $k_{\text {pre }}$. Similarly, if $i=m-1$, then the suffix of position $j+\left|\pi_{c}\right|-1$ in $s_{i}$ (as well as in $\rho_{i}$ ) equals the suffix of position $j_{c}$ in $s$, which is at least $k_{\text {suf }}$; therefore $\rho_{i} \models \pi_{c}$ with suffix at least $k_{\text {suf }}$.

This implies the claim.

We now describe the procedure to verify whether $\Pi \vdash_{\mathrm{d}}$ $\pi$. Let $B$ be the set of all patterns $\pi_{k} \in \Pi$ such that $\pi_{k} \models$ $\pi_{c}$ (and $|B|$ its cardinality). Let $H_{B}$ be the set of all possible matchings of $\pi_{c}$ in patterns of $B$, i.e., $H_{B}$ is the set of all pairs $\left(\pi_{k},[i, j]\right)$ such that $\pi_{k} \in B$ and $\pi_{k}=\pi_{c}$ from $i$ to $j$. These sets can be computed in time polynomial in the size of $\Pi$ and $\pi$; indeed for each pattern $\pi_{k} \in \Pi$, and for each position in $\pi_{k}$, we simply check whether $\pi_{k} \models \pi_{c}$ starting from that position.

We now show that if $B=\emptyset$, then $\Pi \nvdash_{\mathrm{d}} \pi$. Let $<_{\Pi}$ be an arbitrary total order on $\Pi$. We know that there exists a string $s \models_{\mathrm{d}}\left(\Pi,<_{\Pi}\right)$ satisfying conditions of Claim 1. Clearly $s \models_{\mathrm{d}} \Pi$, but on the other hand $s \not \models \pi$, otherwise by Claim 1, we would have $\pi_{i} \models \pi_{c}$ for some $\pi_{i} \in \Pi$. Then $\pi_{i}$ would belong to $B$, which would contradict the fact that $B$ is empty. Hence $\Pi \nvdash_{\mathrm{d}} \pi$ for empty $B$.

Now assume $B \neq \emptyset$. Observe that for each string $s$, we have $s \models_{\mathrm{d}} \Pi$ iff $s \models_{\mathrm{d}}\left(\Pi,<_{B}\right)$ for some total order $<_{B}$ on $B$.

For each total order $<_{B}$ on $B$ and for each matching $\mu=\left(\pi_{k},[i, j]\right) \in H_{B}$, define integers pre ${ }_{<_{B}}(\mu)$ and $\operatorname{suf}_{<_{B}}(\mu)$ as the prefix and suffix of the interval $[i, j]$ in $\mu$, ordered with $<_{B}$. More precisely:

$$
\begin{aligned}
& \operatorname{pre}_{<_{B}}(\mu)=i+\sum_{\pi_{l} \in B, \pi_{l}<B}\left|\pi_{k}\right|, \\
& \text { suf }_{<_{B}}(\mu)=\left|\pi_{k}\right|-j-1+\sum_{\pi_{l} \in B, \pi_{k}<{ }_{B} \pi_{l}}\left|\pi_{l}\right| .
\end{aligned}
$$

Since $H_{B}$ can be computed in polynomial time in the size of $\Pi$ and $\pi$, for each given total order $<_{B}$, the integers $\operatorname{pre}_{<_{B}}(\mu)$ and $\operatorname{suf}_{<_{B}}(\mu)$ for all $\mu \in H_{B}$ can be computed in polynomial time as well.

Claim 2. For each string such that $s \models_{\mathrm{d}}\left(\Pi,<_{B}\right)$ and pattern $\pi_{k} \in B$, there exists a partition $\left(S, S^{\prime}\right)$ of $\Pi \backslash B$ and integers $P \geqslant \sum_{\pi_{i} \in S}\left|\pi_{i}\right|$ and $X \geqslant \sum_{\pi_{i} \in S^{\prime}}\left|\pi_{i}\right|$ such that the following holds:

if $\mu \in H_{B}$ is a matching in $\pi_{k}$, then $s \models \pi_{c}$ with prefix pre $_{<_{B}}(\mu)+P$ and suffix suf ${ }_{<B}(\mu)+X$;

Proof. Since $s \models_{\mathrm{d}}\left(\Pi,<_{B}\right)$, there exist disjoint intervals $\left[i_{k}, j_{k}\right]$ in $s$ which match patterns $\pi_{k}$ of $\Pi$, for $k=0, \ldots$, $n-1$. In particular, among these intervals of $s$, the ones matching patterns of $B$ follow the order induced by $<_{B}$. Therefore since $\pi_{k} \in B$, intervals $\left[i_{r}, j_{r}\right]$ such that $\pi_{r}<_{B}$ $\pi_{k}$ precede $\left[i_{k}, j_{k}\right]$. Similarly intervals $\left[i_{r}, j_{r}\right]$ such that $\pi_{k}<_{B} \pi_{r}$ follow $\left[i_{k}, j_{k}\right]$. Moreover there exists a partition $\left(S, S^{\prime}\right)$ of $\Pi \backslash B$ such that intervals $\left[i_{r}, j_{r}\right]$ with $\pi_{r} \in S$ precede $\left[i_{k}, j_{k}\right]$ and intervals $\left[i_{r}, j_{r}\right]$ with $\pi_{r} \in S^{\prime}$ follow $\left[i_{k}, j_{k}\right]$. Since all those intervals are disjoint, and since the cardinality of $\left[i_{r}, j_{r}\right]$ is equal to $\left|\pi_{r}\right|$, we have $i_{k} \geqslant$ $\sum_{\pi_{r}<B} \pi_{k}\left|\pi_{r}\right|+\sum_{\pi_{r} \in S}\left|\pi_{r}\right|$ and $|S|-j_{k}-1 \geqslant \sum_{\pi_{k}<B} \pi_{r}\left|\pi_{r}\right|+$ $\sum_{\pi_{r} \in S^{\prime}}\left|\pi_{r}\right|$. In particular let
$i_{k}=\sum_{\pi_{r}<B \pi_{k}}\left|\pi_{r}\right|+P \quad$ with $P \geqslant \sum_{\pi_{r} \in S}\left|\pi_{r}\right|$

and

$|s|-j_{k}-1=\sum_{\pi_{k}<B}\left|\pi_{r}\right|+X \quad$ with $X \geqslant \sum_{\pi_{r} \in S^{\prime}}\left|\pi_{r}\right|$.

Let $\mu=\left(\pi_{k},\left[i_{c}, j_{c}\right]\right)$ be a matching in $\pi_{k}$, i.e., $\pi_{k}$ matches $\pi_{c}$ from $i_{c}$ to $j_{c}$. By transitivity of the matching relation, $s$ matches $\pi_{c}$ from $i_{k}+i_{c}$ to $i_{k}+j_{c}$. Since $\pi_{k} \in B$, the prefix of $i_{k}+i_{c}$ is

$i_{k}+i_{c}=\sum_{\pi_{r}<B \pi_{k}}\left|\pi_{r}\right|+P+i_{c}=$ pre $_{<_{B}}(\mu)+P$

and the suffix of $i_{k}+j_{c}$ is

$$
\begin{aligned}
|s| & -1-\left(i_{k}+j_{c}\right) \\
& =|s|-1-j_{k}+j_{k}-\left(i_{k}+j_{c}\right) \\
& =\sum_{\pi_{k}<B}\left|\pi_{r}\right|+X+j_{k}-i_{k}-j_{c} \\
& =\operatorname{suf}_{<_{B}}(\mu)+X .
\end{aligned}
$$

Then $s$ matches $\pi_{c}$ with prefix pre ${ }_{<_{B}}(\mu)+P$ and suffix suf $_{<_{B}}(\mu)+X$, as claimed.

We now distinguish two cases. If $|B| \geqslant k_{\text {pre }}+k_{\text {suf }}+1$, then $\Pi \vdash_{\mathrm{d}} \pi$, as shown below.

Claim 3. If $|B| \geqslant k_{\text {pre }}+k_{\text {suf }}+1$, then $\Pi \vdash_{\mathrm{d}} \pi$.

Proof. Given a string $s$ such that $s \models_{\mathrm{d}} \Pi$ then, as observed above, $s \models_{\mathrm{d}}\left(\Pi,<_{B}\right)$ for some total order $<_{B}$ on $B$. W.l.o.g. let $B$, totally ordered with $<_{B}$, be the sequence $\pi_{0} \ldots \pi_{|B|-1}$ of patterns of $\Pi$ and let $r=k_{\text {pre }}$. We now take the pattern $\pi_{r}$ which must belong to $B$ and have, according to $<_{B}$, exactly $k_{\text {pre }}$ preceding patterns in $B$ and at least $k_{\text {suf }}$ following patterns in $B$. Then we take an arbitrary matching $\mu=\left(\pi_{r},[i, j]\right) \in H_{B}$ and have pre ${ }_{<_{B}}(\mu) \geqslant$ $\left|\pi_{0}\right|+\cdots+\left|\pi_{r-1}\right| \geqslant k_{\text {pre }}$ and $\operatorname{suf}_{<_{B}}(\mu) \geqslant\left|\pi_{r+1}\right|+\cdots+$ $\left|\pi_{|B|-1}\right| \geqslant k_{\text {suf }}$. It follows from Claim 2 that $s \models \pi_{c}$ with prefix at least $k_{\text {pre }}$ and suffix at least $k_{\text {suf }}$. This shows that $s \models \pi$ and concludes the proof of the claim.

Now assume $|B|<k_{\text {pre }}+k_{\text {suf }}+1$. In this case $|B|$ is bounded by a constant that only depends on the number of leading and trailing wildcards in $\pi$ (since $\pi$ is fixed); this allows us to consider all possible total orders $<_{B}$ on $B$. That is, we can check whether $\Pi \vdash_{\mathrm{d}} \pi$ by checking $\left(\Pi,<_{B}\right) \vdash_{\mathrm{d}} \pi$ for each total order $<_{B}$ on $B$. Now we show how to check whether $\left(\Pi,<_{B}\right) \vdash_{\mathrm{d}} \pi$.

Assume again w.l.o.g. that $B$ ordered with $<_{B}$ is given by the sequence $\pi_{0}, \ldots, \pi_{|B|-1}$ of patterns of $\Pi$. We first check in time polynomial in the size of $\Pi$ and $\pi$ whether there exists a matching $\mu \in H_{B}$ such that pre ${ }_{<_{B}}(\mu) \geqslant k_{\text {pre }}$ and $\operatorname{suf}_{<_{B}}(\mu) \geqslant k_{\text {suf }}$. The claim below says that this allows to conclude that $\left(\Pi,<_{B}\right) \vdash_{\mathrm{d}} \pi$.

Claim 4. If there exists a matching $\mu \in H_{B}$ such that pre ${ }_{<_{B}}(\mu)$ $\geqslant k_{\text {pre }}$ and suf $<_{B}(\mu) \geqslant k_{\text {suf }}$, then $\left(\Pi,<_{B}\right) \vdash_{\mathrm{d}} \pi$. 
Proof. Given a string $s$ such that $s \models_{\mathrm{d}}\left(\Pi,<_{B}\right)$, by Claim 2, $s \models \pi_{c}$ with prefix at least pre $_{<_{B}}(\mu) \geqslant k_{\text {pre }}$ and suffix at least $\operatorname{suf}_{<_{B}}(\mu) \geqslant k_{\text {suf }}$. Therefore $s \models \pi$.

If there does not exist a matching satisfying conditions of Claim 4, there are two possibilities covered by the following three claims.

Claim 5. If for all $\pi_{i} \in B$, either all matchings $\mu \in H_{B}$ into $\pi_{i}$ have pre ${ }_{<_{B}}(\mu)<k_{\text {pre }}$ or all matchings $\mu \in H_{B}$ into $\pi_{i}$ have suf $_{<_{B}}(\mu)<k_{\text {suf }}$, then $\left(\Pi,<_{B}\right) \nvdash_{\mathrm{d}} \pi$.

Proof. We show that there must exist a partition of the sequence $\left(\pi_{0}, \ldots, \pi_{|B|-1}\right)$ into two (possibly empty) subsequences $\sigma_{1}=\left(\pi_{0}, \ldots, \pi_{j}\right)$ and $\sigma_{2}=\left(\pi_{j+1}, \ldots, \pi_{|B|-1}\right)$ such that

- for each $\pi_{i}$ in $\sigma_{1}$, all matchings $\mu \in H_{B}$ into $\pi_{i}$ have pre $_{<_{B}}(\mu)<k_{\text {pre }}$ and

- for each $\pi_{i}$ in $\sigma_{2}$, all matchings $\mu \in H_{B}$ into $\pi_{i}$ have suf ${ }_{<B}(\mu)<k_{\text {suf }}$.

Let $j$ be the maximum index in $[0, \ldots,|B|-1]$ such that all the matchings $\mu \in H_{B}$ into $\pi_{j}$ have pre ${ }_{<_{B}}(\mu)<$ $k_{\text {pre }}$, if it exists. If $j$ exists, let $\sigma_{1}=\left(\pi_{0}, \ldots, \pi_{j}\right)$ and $\sigma_{2}=\left(\pi_{j+1}, \ldots, \pi_{|B|-1}\right)$, otherwise let $\sigma_{1}$ be empty and $\sigma_{2}=\left(\pi_{0}, \ldots, \pi_{|B|-1}\right)$. Clearly, for all $\pi_{i}$ in $\sigma_{1}$ and for all matchings $\mu \in H_{B}$ into $\pi_{i}$, we also have pre $<_{B}(\mu)<k_{\text {pre }}$. Moreover, by definition of $\sigma_{1}$ and $\sigma_{2}$ and by the hypothesis of the claim, for all $\pi_{i}$ in $\sigma_{2}$, all matchings $\mu \in H_{B}$ into $\pi_{i}$ have suf $<_{B}(\mu)<k_{\text {suf }}$. This proves the above mentioned properties of $\sigma_{1}$ and $\sigma_{2}$.

Now let $\pi_{\text {pre }}$ (resp., $\pi_{\text {suf }}$ ) be the pattern obtained as the concatenation of patterns of $\sigma_{1}$ (resp., $\sigma_{2}$ ), in the same order as they appear in $\sigma_{1}$ (resp., $\sigma_{2}$ ). We take the totally ordered set of patterns $O=\left\{\pi_{\text {pre }}, \pi_{|B|}, \ldots, \pi_{n-1}, \pi_{s u f}\right\}$ and let $<_{0}$ be the corresponding total order. We know that there exists a string $s$ satisfying conditions of Claim 1 with $O$. Notice that $s \models_{\mathrm{d}}\left(\Pi,<_{B}\right)$; we now show that $s \not \forall \pi$, thus showing that $\left(\Pi,<_{B}\right) \nvdash_{\mathrm{d}} \pi$.

Assume to the contrary that $s=\pi$. Then there exists an index $i \in[0, \ldots,|O|-1]$ where the conditions of Claim 1 are satisfied. This index $i$ must be equal to either 0 or $|O|-1$, otherwise there would exists a pattern in $\left\{\pi_{|B|}, \ldots, \pi_{n-1}\right\}$ matching $\pi_{c}$; then this pattern would belong to $B$, contradicting the hypothesis that $B=$ $\left\{\pi_{0}, \ldots, \pi_{|B|-1}\right\}$. Assume first $i=0$.

In this case, by Claim $1, \pi_{\text {pre }} \models \pi_{c}$ with prefix $p \geqslant k_{\text {pre }}$. On the other hand $p$ must be at most equal to the maximum value of $\operatorname{pre}_{<_{B}}(\mu)$ for all matchings $\mu \in H_{B}$ in patterns of $\sigma_{1}$. By definition of $\sigma_{1}$, this maximum value is strictly less than $k_{\text {pre }}$, then $p<k_{\text {pre }}$. This is a contradiction.

With a symmetric argument we reach a contradiction also in the case that $i=|O|-1$. This proves that $\left(\Pi,<_{B}\right) \nvdash_{\mathrm{d}} \pi$, as claimed.

If conditions of Claim 5 are not satisfied, there must exist $\pi_{r} \in B$ and two matchings $\mu_{1}, \mu_{2} \in H_{B}$ in $\pi_{r}$ such that suf ${ }_{<_{B}}\left(\mu_{1}\right) \geqslant k_{\text {suf }}$ and pre ${ }_{<_{B}}\left(\mu_{2}\right) \geqslant k_{\text {pre. }}$. On the other hand we must have pre ${ }_{<_{B}}\left(\mu_{1}\right)<k_{\text {pre }}$ and suf ${ }_{<_{B}}\left(\mu_{2}\right)<k_{\text {suf }}$, otherwise either $\mu_{1}$ or $\mu_{2}$ would satisfy conditions of Claim 4 .
(Notice that such a $\pi_{r}$, as well as $\mu_{1}$ and $\mu_{2}$ can be found by simply scanning $H_{B}$.) In this situation there are again two cases depending on whether $\sum_{\pi \in \Pi \backslash B}\left|\pi_{i}\right| \geqslant k_{\text {pre }}+k_{\text {suf }}$ holds.

Claim 6. If $\sum_{\pi \in \Pi \backslash B}\left|\pi_{i}\right| \geqslant k_{\text {pre }}+k_{\text {suf }}$, then $\left(\Pi,<_{B}\right) \vdash_{\mathrm{d}} \pi$.

Proof. Let $s \models_{\mathrm{d}}\left(\Pi,<_{B}\right)$, then $s$ with the pattern $\pi_{r}$ satisfies properties of Claim 2. In particular, since both $\mu_{1}$ and $\mu_{2}$ are matchings in $\pi_{r}$, Claim 2 implies that there exist integers $P$ and $X$ such that $P+X \geqslant \sum_{\pi_{i} \in \Pi \backslash B}\left|\pi_{i}\right| \geqslant$ $k_{\text {pre }}+k_{\text {suf }}$ and both the following conditions hold:

- $s \models \pi_{c}$ with prefix $p_{1}=$ pre $_{<_{B}}\left(\mu_{1}\right)+P$ and suffix $x_{1}=$ $\operatorname{suf}_{<_{B}}\left(\mu_{1}\right)+X \geqslant k_{\text {suf }}$;

- $s \models \pi_{c}$ with prefix $p_{2}=$ pre $_{<_{B}}\left(\mu_{2}\right)+P \geqslant k_{\text {pre }}$ and suffix $x_{2}=s u f_{{ }_{B}}\left(\mu_{2}\right)+X$.

Now there are two cases. If $P \geqslant k_{\text {pre }}$, then $p_{1} \geqslant k_{\text {pre }}$, therefore $s=\pi_{c}$ with prefix $p_{1} \geqslant k_{\text {pre }}$ and suffix $x_{1} \geqslant k_{\text {suf }}$. If conversely $P<k_{\text {pre }}$, then $X>k_{\text {suf }}$, hence $x_{2}>k_{\text {suf }}$; therefore $s \models \pi_{c}$ with prefix $p_{2} \geqslant k_{\text {pre }}$ and suffix $x_{2} \geqslant k_{\text {suf }}$. In both cases $s=\pi$. This shows that $\left(\Pi,<_{B}\right) \vdash_{\mathrm{d}} \pi$.

If $\sum_{\pi_{i} \in \Pi \backslash B}\left|\pi_{i}\right|<k_{\text {pre }}+k_{\text {suf }}$, this quantity is a constant depending only on the number of leading and trailing wildcards in the pattern $\pi$. Then we consider all possible partitions of $\Pi \backslash B$ into two sets $S$ and $S^{\prime}$; there is a constant number of them. Checking all these partitions, we can verify whether $\left(\Pi,<_{B}\right) \vdash_{\mathrm{d}} \pi$ as follows.

Claim 7. If for each partition $\left(S, S^{\prime}\right)$ of $\Pi \backslash B$ there exists a matching $\mu \in H_{B}$ into $\pi_{r}$ such that pre ${ }_{<_{B}}(\mu)+\sum_{\pi_{i} \in S}\left|\pi_{i}\right| \geqslant$ $k_{\text {pre }}$ and suf $<_{B}(\mu)+\sum_{\pi_{i} \in S^{\prime}}\left|\pi_{i}\right| \geqslant k_{\text {suf }}$, then $\left(\Pi,<_{B}\right) \vdash_{d} \pi$. Otherwise $\left(\Pi,<_{B}\right) \nvdash_{\mathrm{d}} \pi$.

Proof. Assume $s \models_{\mathrm{d}}\left(\Pi,<_{B}\right)$. Then we know that for $s$ and $\pi_{r}$ there exists a partition $\left(S, S^{\prime}\right)$ of $\Pi \backslash B$ and integers $P$ and $X$ as stated in Claim 2. By the hypothesis, we also know that there exists a matching $\mu \in H_{B}$ into $\pi_{r}$ such that pre $<_{B}(\mu)+\sum_{\pi_{i} \in S}\left|\pi_{i}\right| \geqslant k_{\text {pre }}$ and suf $_{<_{B}}(\mu)+$ $\sum_{\pi_{i} \in S^{\prime}}\left|\pi_{i}\right| \geqslant k_{\text {suf }}$. Then by Claim 2, $s \models \pi_{c}$ with prefix pre $_{<B}(\mu)+P \geqslant$ pre $_{<_{B}}(\mu)+\sum_{\pi_{i} \in S}\left|\pi_{i}\right| \geqslant k_{\text {pre }}$ and suffix suf $_{<_{B}}(\mu)+X \geqslant$ suf $_{<_{B}}(\mu)+\sum_{\pi_{i} \in S^{\prime}}\left|\pi_{i}\right| \geqslant k_{\text {suf }}$. This shows that $s \models \pi$ and therefore $\left(\Pi,<_{B}\right) \vdash_{\mathrm{d}} \pi$.

Conversely, assume there exists a partition $\left(S, S^{\prime}\right)$ of $\Pi \backslash B$ such that all matchings $\mu \in H_{B}$ into $\pi_{r}$ have either pre $_{<_{B}}(\mu)+\sum_{\pi_{i} \in S}\left|\pi_{i}\right|<k_{\text {pre }}$ or suf ${ }_{<_{B}}(\mu)+\sum_{\pi_{i} \in S^{\prime}}\left|\pi_{i}\right|<$ $k_{\text {suf }}$. Notice that in particular $\mu_{1}$ must have pre $<_{B}\left(\mu_{1}\right)+$ $\sum_{\pi_{i} \in S}\left|\pi_{i}\right|<k_{\text {pre }}$, because it satisfies $\operatorname{suf}_{<B}\left(\mu_{1}\right) \geqslant k_{\text {suf }}$. Similarly we must have suf ${ }_{<_{B}}\left(\mu_{2}\right)+\sum_{\pi_{i} \in S^{\prime}}\left|\pi_{i}\right|<k_{\text {suf }}$.

Let $\pi_{S}$ and $\pi_{S^{\prime}}$ be the concatenations of patterns of $S$ and of $S^{\prime}$, respectively, in an arbitrary order. Let $\pi_{S S^{\prime}}$ be $\pi_{S} \pi_{0} \ldots \pi_{|B|-1} \pi_{S^{\prime}}$. We know there exists a string $s$ satisfying the conditions of Claim 1 with $O=\left\{\pi_{S S^{\prime}}\right\}$; notice that $s \models_{\mathrm{d}}\left(\Pi,<_{B}\right)$. We now show that $s \not \models \pi$, thus showing that $\left(\Pi,<_{B}\right) \nvdash_{\mathrm{d}} \pi$.

Assume to the contrary that $s \models \pi$. Then, by Claim 1 , $\pi_{S S^{\prime}}=\pi_{c}$ with prefix $p \geqslant k_{\text {pre }}$ and suffix $x \geqslant k_{\text {suf }}$. Moreover notice that the existence of matchings $\mu_{1}$ and $\mu_{2}$ in 
$\pi_{r}$ implies that $\pi_{S S^{\prime}} \models \pi_{c}$ with prefix $\left|\pi_{S}\right|+$ pre $_{<B}\left(\mu_{i}\right)$ and suffix $\left|\pi_{S^{\prime}}\right|+\operatorname{suf}_{<_{B}}\left(\mu_{i}\right)$, for both $i=1$ and $i=2$. Now there are three cases.

- If $p \leqslant\left|\pi_{S}\right|+\operatorname{pre}_{<_{B}}\left(\mu_{1}\right)$ then $p<k_{\text {pre }}$, which is a contradiction.

- If $x \leqslant\left|\pi_{S^{\prime}}\right|+\operatorname{suf}_{<_{B}}\left(\mu_{2}\right)$ then $x<k_{\text {suf }}$, which is a contradiction.

- Otherwise the matching into $\pi_{S S^{\prime}}$ with prefix $p$ and suffix $x$ corresponds to some matching into $\pi_{r}$. More precisely there exists a matching $\mu \in H_{B}$ into $\pi_{r}$ such that $p=\left|\pi_{S}\right|+$ pre $_{<_{B}}(\mu)$ and $x=\left|\pi_{S^{\prime}}\right|+\operatorname{suf}_{<_{B}}(\mu)$. Then either $p<k_{\text {pre }}$ or $x<k_{\text {suf }}$. This is also a contradiction.

This proves that $s \not \models \pi$ and therefore $\left(\Pi,<_{B}\right) \nvdash_{\mathrm{d}} \pi$, thus concluding the proof of the claim.

Since one of the cases considered in Claims 3, 4, 5, 6 and 7 has to occur, the above results define a procedure for checking whether $\Pi \vdash_{d} \pi$.

The cost of this procedure is dominated by the cost of the following computation:

- compute sets $B$ and $H_{B}$;

- check $|B| \geqslant k_{\text {pre }}+k_{\text {suf }}+1$;

- in the case $|B|<k_{\text {pre }}+k_{\text {suf }}+1$, for each total order $<_{B}$ on $B$

- compute pre ${ }_{<_{B}}(\mu)$ and suf $_{<_{B}}(\mu)$ for all $\mu \in H_{B}$;

- check conditions of Claims 4, 5, 6 and 7.

We have already observed that sets $B$ and $H_{B}$, as well as pre $_{<_{B}}(\mu)$ and $\operatorname{suf}_{<_{B}}(\mu)$, for all $\mu \in H_{B}$, can be computed in polynomial time in the sizes of $\Pi$ and $\pi$. Moreover, conditions of Claims 4, 5, 6 can be checked in time polynomial in the sizes of $\Pi$ and $\pi$. This is also true for Claim 7 if $k_{\text {pre }}$ and $k_{\text {suf }}$ are fixed (which is our case, as $\pi$ is fixed). This implies that the cost of the above computation is $O(p(|\Pi|+|\pi|))$, for some polynomial $p$ depending only on the number of leading and trailing wildcards in $\pi$, and concludes the proof of Theorem 1 .

Remark. The proof shows that the problem of checking whether $\Pi \vdash_{\mathrm{d}} \pi$, with $\pi$ being a part of the input, is in PTIME if the number of leading and trailing wildcards in $\pi$ is fixed. That is, for every fixed $k, \ell$, the problem of checking whether $\Pi \vdash_{\mathrm{d}}\left({ }_{-}\right)^{k} a \pi b\left({ }_{-}\right)^{\ell}$, with $a, b \in \mathcal{L}$ and $\pi \in \mathcal{L}^{*}$, is solvable in polynomial time when both $\Pi$ and $\pi$ are inputs.

\section{Acknowledgement}

Supported by EPSRC grants E005039 and G049165 and by the FET-Open FOX project, grant agreement 233599. We thank Pablo Barceló and Gonzalo Navarro for their comments.

\section{References}

[1] S. Abiteboul, L. Segoufin, V. Vianu, Representing and querying XML with incomplete information, ACM TODS 31 (2006) 208-254.

[2] P. Barceló, L. Libkin, A. Poggi, C. Sirangelo, XML with incomplete information: Models, properties, and query answering, in: PODS'09, pp. 237-246.

[3] H. Björklund, W. Martens, T. Schwentick, Conjunctive query containment over trees, in: DBPL'07, pp. 66-80.

[4] D. Maier, The complexity of some problems on subsequences and supersequences, J. ACM 25 (2) (1978) 322-336.

[5] Document Object Model (DOM). W3C Recommendation, April 2004, http://www.w3.org/TR/DOM-Level-3-Core. 